\title{
Finales en sandhi en aquitanopyrénéen Langue de France - Occitan
}

\author{
Vincent Rivière \\ Chargé de mission linguistique, Congrès Permanent de la Langue Occitane, Billère \\ Membre associé, Axe VAst, CLLE-ERSS UMR 5263, Université Toulouse Jean Jaurès,Toulouse \\ v.riviere@locongres.org
}

\section{Résumé}

La forme dels/deus est réalisée indistinctement [des] en occitan dans la zone toulousaine, alors que nous trouvons la forme [del] au singulier côté languedocien et [du] côté gascon. Je pose l'hypothèse suivante : la structure syllabique va conditionner cette réalisation dans le sens où la permissivité de la syllabe dans cette zone ne laisse qu'une position dans laquelle le morphème du pluriel est prioritaire. Gascon oriental et languedocien occidental relèvent d'un modèle où il faut clairement admettre que la finale dépasse la structure syllabique régulière. Cela peut s'expliquer d'une part par des syllabes à noyaux nuls, d'autre part par une conception récursive de la syllabe qui peut être schématisée de la façon suivante $: \operatorname{mot}=(\text { syllabe })^{*+}$ (Consonne facultative). Cela rejoint la formule proposée pour le français dans Dell (1995). Certaines finales sont ainsi des attaques d'une syllabe à noyau nul :
a) [l'up] : (lu) $(\mathrm{p} \emptyset)$
b)
$\left[\mathrm{s}^{\prime} \varepsilon r p\right]:(\mathrm{s \varepsilon r})(\mathrm{p} \emptyset)$

L'hypothèse du noyau nul explique que la particule excédentaire soit une attaque. Il faut cependant limiter les syllabes $(C \emptyset)$ aux finales : sèrps [s'हrs] $=(\mathrm{s} \varepsilon r)(\mathrm{s} \emptyset)$. Le morphème du pluriel $/ \mathrm{s} /$ occupera prioritairement la position d'attaque en finale à la place des autres consonnes. Ce mécanisme sera appliqué à la contraction préposition - article.

\begin{abstract}
Finals in sandhi in aquitanopyrenean - France language : Occitan. The form dels / deus is realized indistinctly in Occitan of Toulouse, while we find the singular form [del] in the Languedoc area and [du] in the Gascon area. Here is my hypothesis : the syllabic structure will determine this realization in the sense that the permissiveness of the syllable in this zone leaves only one position in which the morpheme of the plural is more important. Eastern gascon and western languedocian belong to a model where it must be clearly admitted that the finale exceeds the regular syllabic structure. This can be explained on the one hand by syllables with null nuclei, on the other hand by a recursive conception of the syllable which can be schematized as follows: word $=(\text { syllable })^{*+}$ (optional Consonant $)$. This joins the formula proposed for French in Dell (1995). Some finals are thus attacks of a kernel-zero syllable :
\end{abstract}
a) [l'up] : (lu $)(\mathrm{p} \emptyset)$
b)

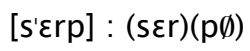


The null nucleu hypothesis explains that the excess particle is an attack. However, the syllables $(\mathrm{C} \emptyset)$ must be limited to the finals: sèrps - [s'Ers] = $(s \varepsilon r)(s \emptyset)$. The morpheme of the plural / s / will promptly occupy the attack position in the final instead of the other consonants. This mechanism will be applied to the contraction preposition - article.

Suite à différents collectages en gascon oriental et languedocien occidental (dans la zone de l'article le [le]), Gers et Ariège notamment, complétés par une consultation des atlas linguistiques, je me suis interrogé, dans un premier temps, sur les formes des contractions de l'article toulousain le au pluriel, avec la préposition de [de] $(d e+l e)$. La forme dels/deus (des) réalisée indistinctement [des] ([dej]), alors qu'elle se réalise [del] au singulier côté languedocien et $[\mathrm{du}]$ côté gascon, a particulièrement attiré mon attention. En effet, nous aurions pu attendre les réalisations [dels] et [dus]. Les zones dans lesquelles j'ai eu l'occasion de collecter comportant à la fois l'article le et la contraction [des] ([dej]), j'ai posé trop hâtivement l'hypothèse suivante selon laquelle la forme contractée [des] était spécifiquement associée à l'emploi de l'article [les] typique de la région toulousaine.

(1) Synthèse article défini : Carte ALF 285 - le ciel

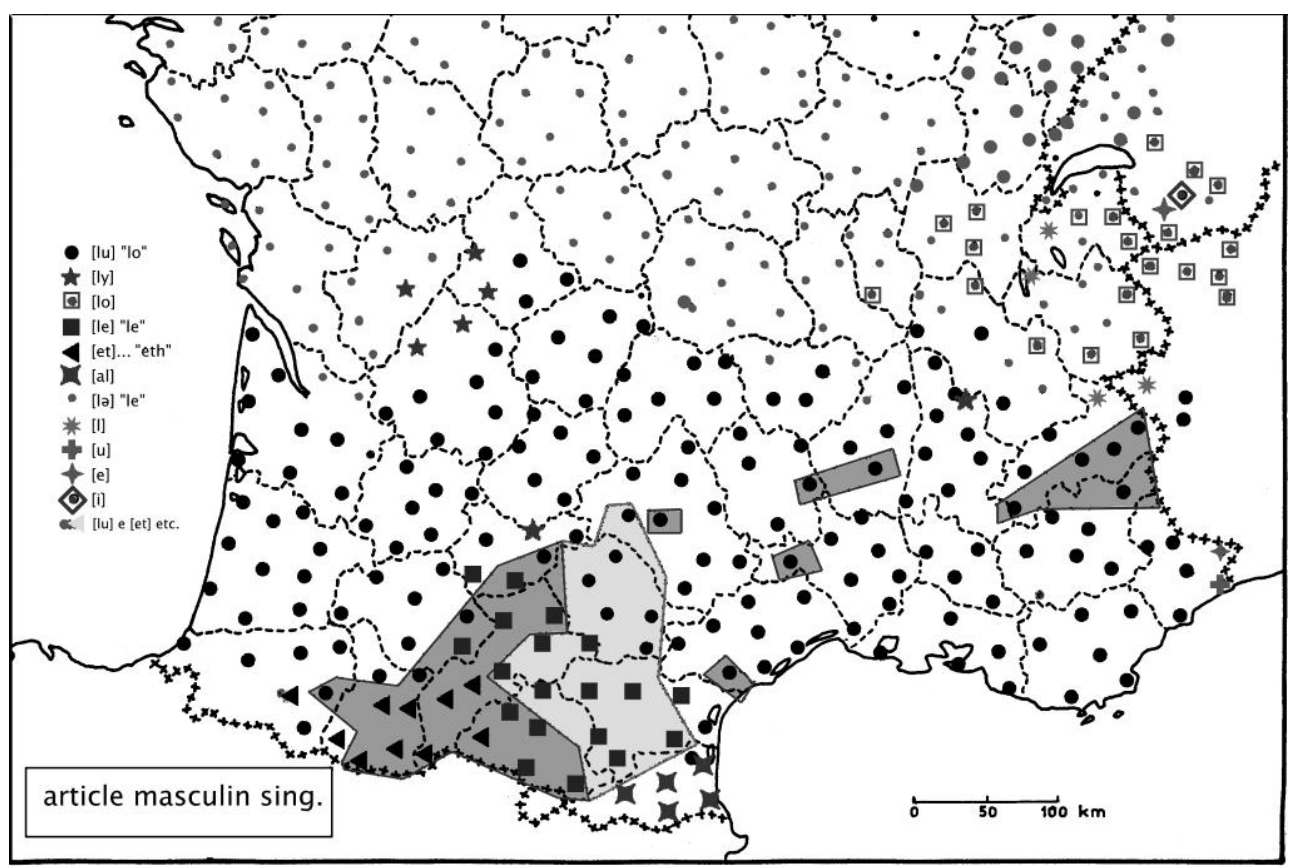

G. Brun-Trigaud (CNRS-BCL, Nice, UMR 7320), ALF 285 - le ciel, à partir des données de $\mathrm{P}$. Sauzet, - OC 401- Lingüistica

Légende de la figure (1):
[des]
[dej]
- $[\mathrm{lu}] \ll 1 \mathrm{lo} »$
- $[\mathrm{le}] \ll \mathrm{le} »$
- [et]... « eth »

Mon investigation m'a permis de constater que la réalisation [des] ([dej]) était présente sur un espace géographique plus large que celui de l'article [le], notamment au nord de 
Toulouse en zone de l'article lo, plus au sud en zone de l'article eth. L'extrait de la carte 285 « le ciel» de l'Atlas linguistique de la France (ALF) adaptée par G. Brun à partir des données de P. Sauzet dans le cadre de son cours de linguistique OC 401 à l'Université de Toulouse 2 Jean Jaurès, met à jour la présence de toutes les formes de l'article défini en occitan. J'ai superposé à cette carte les zones de contraction [des] ([dej]), et ai constaté que mon hypothèse était bien caduque : la contraction plurielle [des] ([dej]) ne correspond pas à la zone de l'article le.

La variation des finales en occitan est dépendante du format syllabique, propre à une aire géolinguistique, tel que la définit Sauzet (2004). Ainsi, les finales de l'aquitanopyrénéen (AP), mises en exergue par Bec (1963), excèderont le format d'une syllabe régulière comme le décrit Dell (1995) pour le français. Je m'appuierai donc sur la syllabe à noyau nul pour expliquer les combinaisons segmentales dans le cadre de la phonologie du gouvernement développée par Kaye \& Lowenstamm (1984). L'étude des finales en gascon oriental, et par extension du languedocien occidental, soit l'Est de l'AP, mettra en lumière les codas légitimes de cette zone. Il apparaîtra que le morphème consonantique du pluriel est prioritaire au travers l'étude de l'article le au pluriel en enclise.

Je pose donc l'hypothèse suivante : la structure syllabique va conditionner la réalisation [des] dans le sens où la permissivité de la syllabe dans cette zone ne laisse qu'une position de coda possible en sandhi dans laquelle le morphème du pluriel est prioritaire. Le morphème de définitude [1] disparaîtra au profit de ce dernier. Un continuum existe ainsi, notamment en sandhi au pluriel, étendu au-delà de la forme de l'article défini le et des isoglosses séparant les dialectes gascon et languedocien. Cet argument amènera une notion supplémentaire de graduation de tolérance des finales au sein même de l'aquitanopyrénéen, d'Est en Ouest.

\section{Classification dialectale}

Bec (1963) définit trois grands ensembles au sein de la langue occitane sur la base de la tolérance des finales: l'arverno-méditerranéen qui conserve très peu les finales, l'aquitanopyrénéen qui les conserve régulièrement, et l'occitan central qui est un intermédiaire. Sumien (2009) met en valeur cette classification dans la carte suivante. 


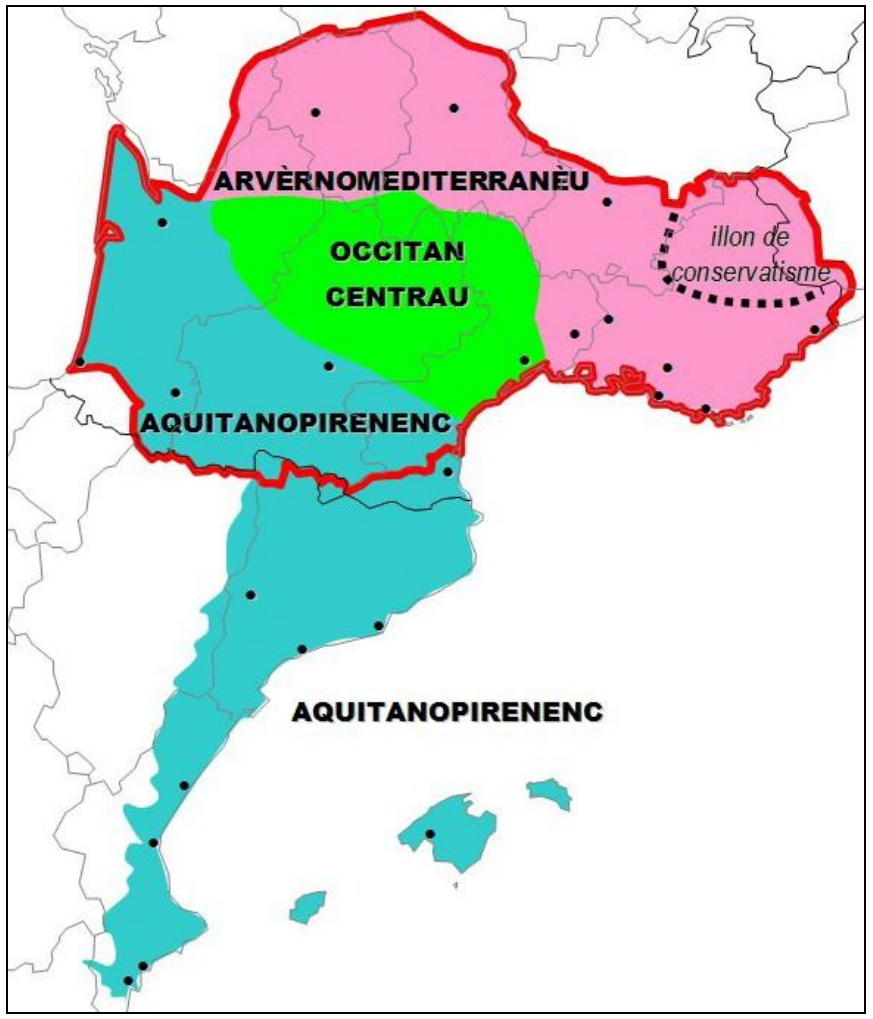

Sumien (2009)

Le tableau suivant permettra, à partir d'exemples, d'établir le lien avec la classification dialectale du diasystème proposé par Bec (1973), régulièrement utilisée aujourd'hui.

(1) Variation des finales occitanes

\begin{tabular}{|c|c|c|c|}
\hline & sal & lop & sèrp \\
\hline $\begin{array}{l}\text { Gascon oriental } \\
\text { (G.or) }\end{array}$ & [s'aw] & [l'up] & [s'erp] \\
\hline $\begin{array}{l}\text { Languedocien } \\
\text { occidental (L.occ) }\end{array}$ & [s'al] & [l'up] & [s'\&rp] \\
\hline $\begin{array}{l}\text { Languedocien } \\
\text { oriental (L.or) }\end{array}$ & [s'al] / [s'aw] & [l'up] & [s'Er] \\
\hline Provençal & [s'aw] & [l'u] & {$\left[s^{\prime} \varepsilon r\right]$} \\
\hline
\end{tabular}

A partir de Sauzet (2004)

La première colonne comporte une sonante en coda, régulièrement présente ([1] ou réalisée vocalisée $[\mathrm{w}]$ ) à travers les dialectes. Les occlusives maintenues en position finale en gascon oriental et languedocien occidental s'opposent à l'effacement systématique du 
provençal (représentatif de l'arverno-méditerranéen), alors que le languedocien oriental présente une situation intermédiaire : conservation après voyelle, effacement après élément non syllabique.

Sauzet (2004) propose une structure syllabique pour le languedocien occidental qui correspond parfaitement au schéma du gascon oriental synthétisée comme suit: G.or et L.occ relèvent d'un modèle où il faut clairement admettre que la finale dépasse le format syllabique régulier (les syllabes intérieures étant supposées régulières). Cela peut s'expliquer d'une part par des syllabes à noyaux nuls, d'autre part par une conception récursive de la syllabe qui peut être schématisée de la façon suivante, où l'astérisque note la répétition illimitée du constituant :

(2) Mot en aquitanopyrénéen

$$
\text { mot }=(\text { syllabe })^{*+}(\text { Consonne facultative })
$$

Cela rejoint la formule proposée pour le français dans Dell (1995). Certaines finales sont ainsi des attaques d'une syllabe à noyau nul :

(3) Les consonnes finales comme attaques d'une syllabe à noyau nul
a)
[l'up] : $(\mathrm{lu})(\mathrm{p} \emptyset)$
b) $\quad\left[s^{\prime} \varepsilon r p\right]:(\mathrm{ser})(\mathrm{p} \emptyset)$

L'hypothèse du noyau nul explique que la particule excédentaire soit une attaque. Cela assure que la particule qui s'ajoute à la séquence des syllabes canoniques est la première partie d'une syllabe bien formée, toute syllabe canonique en occitan étant supposée [C(c)VC] selon Sauzet (1993). Dans l'approche de Kaye \& Lowenstamm (1984), un segment non-syllabique final est nécessairement attaché à une position $\mathrm{C}$ que suit un $\mathrm{V}$ sans contenu, il forme donc une attaque suivie d'un noyau nul. La phonologie du gouvernement parvient à une unification de la légitimation interne et finale puisque la finale entre dans le format général en formant une syllabe $\mathrm{CV}$, mais la particularité d'avoir un noyau nul ne l'isole pas. Le mécanisme de légitimation pour une syllabe à noyau nul doit, ceci étant, être limité pour éviter une suite segmentale incohérente : il faut donc limiter les syllabes (CØ) aux finales.

(4) Légitimation du noyau nul limité aux finales

Nous aurons donc au pluriel la forme suivante :

$$
\text { sèrps - [s'عrs] : (sعr) }(s \emptyset)
$$

Le morphème du pluriel /s/ occupera prioritairement et systématiquement la position d'attaque en finale à la place des autres consonnes $(/ \mathrm{p} /, / \mathrm{k} /$, etc. $)$.

\section{Les finales du gascon oriental}

Hormis après affriquée sourde, le morphème du pluriel est ajouté soit directement au lexème, soit après insertion vocalique. En contexte d'occlusive finale, le pluriel se formera systématiquement par l'affriquée sourde [ $t \underline{\underline{S}}$ ].

(5) Finales simples du gascon oriental au pluriel 


\begin{tabular}{|c|c|c|c|c|}
\hline $\begin{array}{c}\text { marque du } \\
\text { pluriel }\end{array}$ & $\begin{array}{l}\text { finale } \\
\text { phonologique }\end{array}$ & $\begin{array}{l}\text { schéma } \\
\text { phonologique }\end{array}$ & $\begin{array}{l}\text { pluriel } \\
\text { phonétique }\end{array}$ & $\begin{array}{l}\text { pluriel } \\
\text { graphique }\end{array}$ \\
\hline \multirow{6}{*}{$\begin{array}{c}\text { occlusive }+\mathrm{s}= \\
\text { ts }\end{array}$} & $-p$ & $\backslash k a p \backslash$ & [k'ats] & caps \\
\hline & $-b$ & ||$u b \backslash$ & [l'uts] & lops \\
\hline & $-\mathrm{t}$ & \gat \} $&{\text { [g'ats] }} &{\text { gats }} \\
{\hline} &{-d} &{\backslash \text { pulid } \backslash} &{\text { [pul'its] }} &{\text { polits }} \\
{\hline} &{-\mathrm{k}} &{\text { \armanak\} } &{\text { [arman'ats] }} &{\text { armanhacs }} \\
{\hline} &{-\mathrm{g}} &{\backslash p \varepsilon g \backslash} &{\text { [p'cts] }} &{\text { pècs }} \\
{\hline \multirow{4}{*}{\begin{array}{c}\text { obstruante } \\
\text { phonologique }+ \\
\text { es }\end{array}}\begin{array} { c } { \text { obstruante } } \\
{ \text { phonologique } + } \\
{ \text { es } } \end{array}} &{\text {-ts }} &{\mid \text { puts } \mid} &{\text { [p'uzes] }} &{\text { potzes }} \\
{\hline} &{-\int} &{\mid p e \int \backslash} &{\text { [p'efes] }} &{\text { peishes }} \\
{\hline} &{-\mathrm{z}} &{\backslash \text { predz } \backslash} &{\text { [pr'ezes] }} &{\text { preses }} \\
{\hline} &{-\mathrm{s}} &{\mid \text { gros } \backslash} &{\text { [gr'oses] }} &{\text { gròsses }} \\
{\hline \text { invariable }} &{-\mathrm{t} 5} &{\mid \text { papat_| }} &{\text { [pap'at]] }} &{\text { papach }} \\
\hline\end{array}$
\end{tabular}

Le tableau des pluriels en finale complexe qui suit démontre l'importance du morphème consonantique du pluriel.

(6) Finales complexes du gascon oriental au pluriel

\begin{tabular}{|c|c|c|c|c|c|}
\hline \multicolumn{4}{|c|}{$\mathrm{Z}_{\text {nombre }}$} & singulier & pluriel \\
\hline \multirow{6}{*}{$/ p /$} & \multicolumn{3}{|c|}{ réalisation phonétique } & [p] & [ts] \\
\hline & \multirow{2}{*}{ exemple } & \multicolumn{2}{|c|}{ graphique } & lop & lops \\
\hline & & \multicolumn{2}{|c|}{ phonétique } & [lup] & [luts] \\
\hline & après : & \multicolumn{2}{|c|}{ réalisation } & [p] & [s] \\
\hline & \multirow{2}{*}{$\begin{array}{l}\text { liquide ou } \\
\text { semi-cons. }\end{array}$} & \multirow[t]{2}{*}{ ex } & graphique & sèrp & sèrps \\
\hline & & & phonétique & [s'erp] & [s'ers] \\
\hline \multirow{2}{*}{$/ t /$} & \multicolumn{3}{|c|}{ réalisation phonétique } & [t] & [ts] \\
\hline & exemple & \multicolumn{2}{|c|}{ graphique } & gat & gats \\
\hline
\end{tabular}




\begin{tabular}{|c|c|c|c|c|c|}
\hline & & \multicolumn{2}{|c|}{ phonétique } & [gat] & [ts] \\
\hline & \multirow{3}{*}{$\begin{array}{l}\text { après : } \\
\text { liquide ou } \\
\text { semi-cons. }\end{array}$} & \multicolumn{2}{|c|}{ réalisation } & {$[\mathrm{t}]$} & [s] \\
\hline & & \multirow{2}{*}{ ex } & graphique & hèit & hèits \\
\hline & & & phonétique & [hejt] & [hejs] \\
\hline \multirow{6}{*}{$/ \mathrm{k} /$} & \multicolumn{3}{|c|}{ réalisation phonétique } & [k] & [ts] \\
\hline & \multirow[t]{2}{*}{ exemple } & \multicolumn{2}{|c|}{ graphique } & pèc & pècs \\
\hline & & \multicolumn{2}{|c|}{ phonétique } & [pek] & [pets] \\
\hline & après : & \multicolumn{2}{|c|}{ réalisation } & {$[\mathrm{k}]$} & [s] \\
\hline & \multirow{2}{*}{$\begin{array}{l}\text { liquide ou } \\
\text { semi-cons. }\end{array}$} & \multirow[t]{2}{*}{ ex } & graphique & pòrc & pòrcs \\
\hline & & & phonétique & [pork] & [pors] \\
\hline
\end{tabular}

Le tableau permet de visualiser la réalisation récurrente en [t. $]$. Sur les trois occlusives, qu'elles soient seules en finale ou après liquide ou semi-consonne, elles sont réalisées [t: ], ou effacées. L'effacement et la formation de l'affriquée ont en commun de permettre de n'avoir qu'une consonne extrasyllabique, c'est une consonne complexe, mais unique.

Pour mieux comprendre cette récurrence, il faut dans un premier temps étudier l'articulation de chaque consonne et tâcher de comprendre quelles sont les caractéristiques qui sont communes au morphème du pluriel [s]. Les phones [ $\mathrm{t}]$ et [s] sont tous les deux coronaux, l'un dental et l'autre apico-alvéolaire, donc les deux points d'articulation sont très proches. L'affriquée [ $\mathrm{t} \underline{\mathrm{S}}$ ] a donc forcément des caractéristiques communes aux deux, et on comprend aisément que la combinaison dans l'ordre indiqué donne ce résultat. En regardant les caractéristiques des deux autres consonnes, nous relevons bien qu'elles ne sont pas coronals, ce qui engendrerait une articulation beaucoup plus complexe - voire impossible - expliquant donc la convergence vers [ts].

Les exemples sont monosyllabiques, ainsi chaque exemple permet d'étudier, dans une certaine mesure, le format syllabique, et d'essayer directement d'en identifier les codas. La contrepartie en est que, comme il n'y a forcément pas de syllabe intérieure, les hypothèses que nous avançons nécessitent forcément d'autres exemples pour les confirmer.

J'ai d'abord d'identifié les affriquées et certaines occlusives comme coda. Dans le mot lop [lup] ou lops [luts], comme il s'agit d'une rime simple, cela semble possible. Le problème est que nous ne trouvons pas l'équivalent en syllabe intérieure. La solution semble donc de les mettre en position extrasyllabique. Mais en proposant cela, il est difficile d'expliquer que [k] de trauc [trawk] s'efface alors que, lorsqu'il est après rime simple, comme dans pèc [pek], il s'assimile. Sauzet (2004) propose que les occlusives puissent être en coda seulement en finale. Cela explique le paradigme du languedocien oriental suivant : pòrc [por], mais sec [sek] et non [se] comme en provençal. Cela explique également l'asymétrie en liaison du languedocien occidental et du gascon oriental. 
La difficulté est de savoir pourquoi il y a occlusive en finale et non ailleurs. Il propose que nous puissions, dans certaines conditions, trouver une "petite syllabe» - $(\mathrm{C} \varnothing)_{\sigma}-$ une consonne suivie d'un noyau nul. C'est le principe de la récursivité.

Nous pourrions avoir ainsi : ( ( s $)_{\mathrm{A}}\left((\mathrm{e})_{\mathrm{N}}\left((\mathrm{k} \varnothing)_{\sigma}\right)_{\mathrm{C}}\right)_{\sigma}$ pour [sek]. On peut ajouter à cela que si le noyau nul de la "petite syllabe » est placé devant l'attaque d'une autre syllabe dans son domaine phonologique, cela formera une géminée et cette attaque sera ainsi assimilée dans la géminée. Cela expliquerait que nous ayons des occlusives en coda seulement en syllabe finale, mais pas en syllabe intérieure ni en liaison.

Nous pouvons ainsi poser le schéma suivant :

(7) Schéma syllabique de l'aquitanopyrénéen

$$
\begin{aligned}
& {\left[[\mathrm{s}]_{\mathrm{A}}\left[[\varepsilon]_{\mathrm{N}} \mathrm{r}\right]_{\mathrm{C}}\right]_{\sigma} \mathrm{s}_{\mathrm{es}}(\mathrm{A}: \text { attaque, } \mathrm{N}: \text { noyau, } \mathrm{C}: \text { coda, es : extrasyllabique })^{\mathrm{i}}} \\
& (\mathrm{s} \varepsilon \mathrm{r})_{\sigma} \mathrm{p}_{\mathrm{es}}+\mathrm{s} \rightarrow(\mathrm{s} \varepsilon \mathrm{r})_{\sigma} \mathrm{s}_{\mathrm{es}}
\end{aligned}
$$

Le schéma ci-dessus montre la position extrasyllabique réelle, réalisée [s]. Il montre également la position extrasyllabique du singulier, seulement à titre indicatif, pour montrer le passage de l'un à l'autre.

On peut donc se demander pourquoi [t] est conservé dans l'affriquée dans d'autres contextes. Le contexte de proximité de lieu d'articulation semble un argument raisonnable, phénomène que l'on retrouve en languedocien comme l'indiquent Lieutard (2004 -1) et Chabbert (1959). De fait l'affriquée, bien que formée initialement par deux éléments, n'occupe ici qu'une position, celle de extrasyllabique, dans laquelle le morphème du pluriel est bien présent.

\section{L'article défini toulousain Le}

Bien que la voyelle de l'article le [le] ne joue pas un rôle particulier, comparativement aux deux autres articles, dans la réalisation de cette contraction, l'étude de ce dernier au pluriel en enclise apporte un élément phonétique fondamental.

Toulouse, dès avant le milieu du $\mathrm{XIV}^{\mathrm{e}}$ s., l'oblique le, surtout au pl. les employé concurremment avec lo, los. [...] Le tipe masc. le, pl. les s'établit en carc. laur. toul. aqor., [...] Ronjat $(1937,108)$

Carc.laur.toul ${ }^{\text {ii. }}$. le, f. la, plur. M. les / lei, f. las / lai. La limite E. entre ce tipe et le tipe bit. Narb donne à $l e$ l'arrondissement de Castelnaudari, [...]. L'altern. $-s$ devant consonne dure ou voy., $-i$ devant consonne molle est assez ancienne, mais elle n'a pas partout la régularité qu'on attendrait et qu'indiquaient certains exposés trop schématiques. Ronjat $(1937,117)$

Comme en languedocien toulousain, l'article défini le est présent dans le gascon étudié. Cette forme d'article défini est une des caractéristiques principales de ce parler, quand la majorité de la langue occitane emploie l'article lo [lu]. Il est présent aussi bien comme article que comme pronom objet. L'article de Nègre 1978 et le mémoire de Roque-Ferrier 1879 décrivent la zone géographique de présence de ce dernier. 


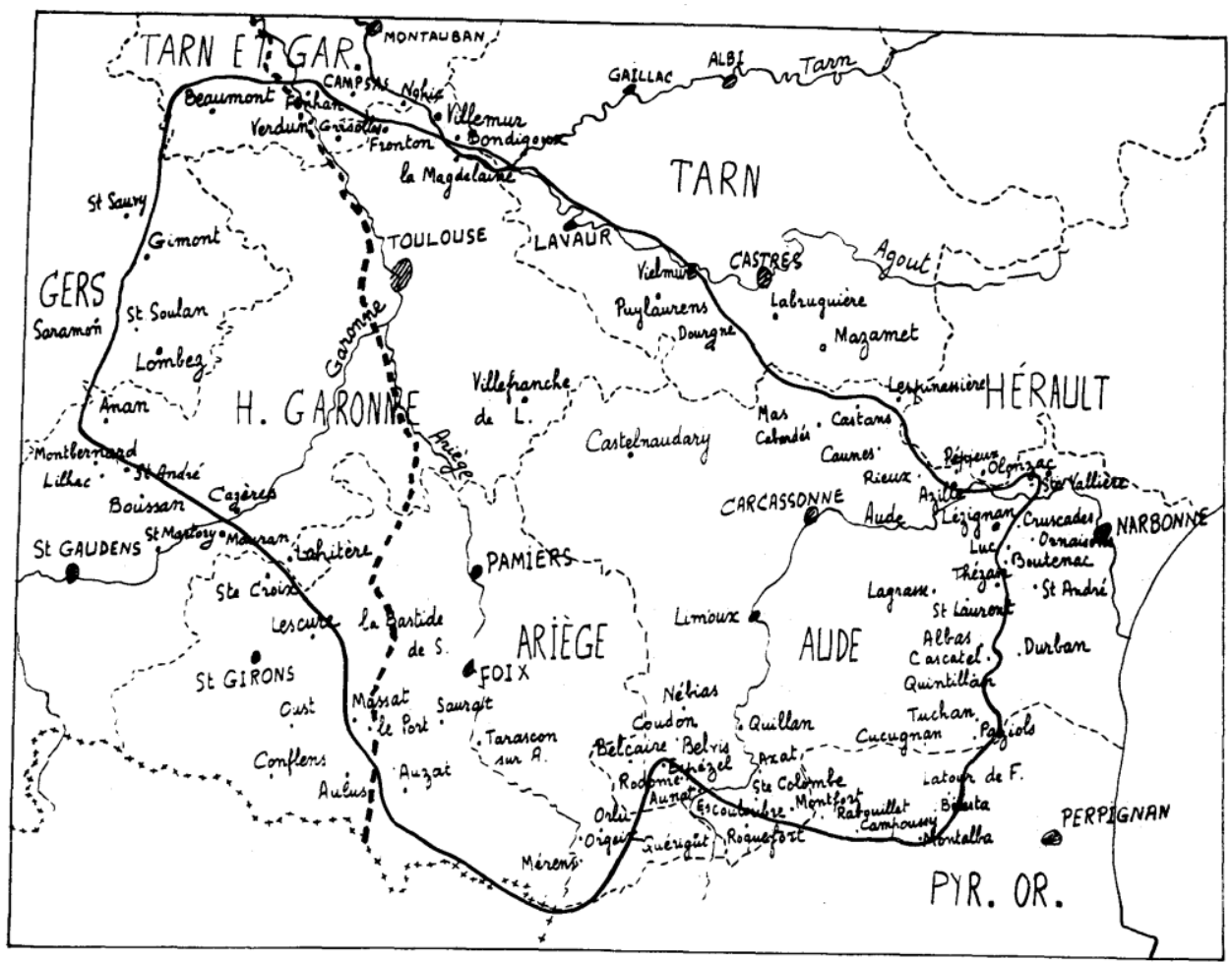

Nègre $(1978,963)$

La zone de l'article le est délimitée par le trait continu épais englobant une grande partie des départements de la Haute-Garonne, de l'Ariège et de l'Aude.

L'analyse du traitement de l'article le côté languedocien, notamment dans les vallées de la Garonne et de l'Ariège, met en évidence une différence importante avec le gascon toulousain. En nous appuyant sur le mémoire de Roque-Ferrier 1879 et sur les enregistrements que j'ai réalisés en Ariège en 2006, à Saurat, nous pouvons relever des contractions spécifiques après que [ke].

Las Pouésios bariados de Daveau, de Carcassonne, l'un des plus brillants poëtes du Languedoc, donnent après une voyelle, et par conséquent semblables de position et d'appui, à ceux de la chanson à Dona Clamença, deux exemples de l'article pluriel és. On le trouve dans une ode sur le passage de la mer Rouge:

Sap pas qu'él fa toumba lés latches goubernaires

Coumo toumbo lé blat joux l'oulan das ségaïres?

Qu'és cèdres dal Liban dabant él soun toumbats?

Et tandis qu'és souldats dé fatigo ranquéjoun,

Lé poplé d'Abraham dins Suèz és dintrat. Roque-Ferrier $(1879,122-123)$

La notation de Daveau est phonétisante et ne correspond pas à la notation orthographique moderne, et est, aujourd'hui, linguistiquement discutable. Les vers de Daveau sont écrits en graphie classique actuelle de la façon suivante:

Sap pas qu'el fa tombar les laches governaires

Coma tomba le blat jos l'olam dals segaires? 
Que'ls cèdres dal Liban davant el son tombats?

E tandis que'ls soldats de fatiga ranquejan,

Le pòble d'Abraam dins Suez es dintrat.

L'analyse est qu'il ne s'agit pas de l'article $e l$, mais d'un cas d'enclise syllabique de l'article sur une conjonction de subordination : [kes] n'est pas $k+e(l) s$, mais bien $k e+(l) s$, et la meilleure preuve est que, quand il n'y a pas d'enclise, il y a la forme les [les].

La forme au pluriel change dans ce contexte. De fait, nous constatons que la consonne phonologique de l'article le au pluriel est effacée et que le morphème consonantique du pluriel -s est conservé. Ferrier établit notamment una similitude de traitement avec l'article pyrénéen eth/eths. Cet usage est assez classique en languedocien.

L'article proclitic s'elidís davant las vocalas : l'òme, l'ora, [...] L'article enclitic masculin pèrd tanben sa vocala, lo singular davant consonanta e lo plural indistinctament davant vocala e consonanta : caça'l gos, caça'ls gosses, [...] Alibert $(2000,73)$

On a vu que les composés avec prép. dans lesquels l'article perd sa voy. sont particulièrement nombreux [...] Toul. festeja-s felibres "fêter les félibres ", fa-s adius "faire ses adieux ", pendent que-l foc..., dempèi que-l mounde es mounde, foro-s plasés « en dehors des plaisirs » [...] Ronjat $(1937,121)$

En languedocien occidental, l'article défini au pluriel en enclise est donc régulièrement réduit au morphème du pluriel. Cette réduction sera similaire en contraction en sandhi.

\section{Gradualité de tolérance croissante d'Est en Ouest au sein de l'aquitanopyrénéen}

Le statut d'un substantif et d'un article (d'une préposition et d'une contraction préposition - article) est sémantiquement et syntaxiquement différent, du fait que l'article est dépendant d'un substantif postposé pour exister. Ainsi, l'article ou la contraction préposition - article seront forcément construits avec des syllabes canoniques, étant donné que l'attaque du mot suivant obligatoire confisquera la position potentielle d'une particule excédentaire. Il n'y aura donc qu'une position possible en coda, et le morphème du pluriel sera prioritaire. En gascon, la forme deu $[\mathrm{du}](\mathrm{de}+\mathrm{le})$ est la vocalisation, réduite à posteriori de $[\mathrm{del}]>[\mathrm{dew}]>[\mathrm{du}]$. Phonologiquement, nous avons bien le phonème $/ 1 /$ dans l'article [du]. Il ne peut donc être conservé, le morphème du pluriel étant prioritaire dans ce parler.

Nous aurons ainsi la répartition suivante :

(8) Réalisation en sandhi à l'Est de l'aquitanopyrénéen

\begin{tabular}{|l|l|l|l|}
\hline $\begin{array}{l}\text { Article défini } \\
\text { singulier }\end{array}$ & $\begin{array}{l}\text { Article défini }+ \\
\text { morphème pluriel }\end{array}$ & $\begin{array}{l}\text { Contraction au } \\
\text { singulier }\end{array}$ & $\begin{array}{l}\text { Contraction }+ \\
\text { morphème pluriel }\end{array}$ \\
\hline le $[\mathrm{le}]$ & les $[\mathrm{les}]$ & G.or $:$ deu $[\mathrm{du}]$ & deus \\
\hline
\end{tabular}




\begin{tabular}{|l|l|l|l|}
\hline & & L.occ : del [del] & dels \\
\cline { 1 - 3 } lo [lu] & los [lus] & del [del] \\
[des]
\end{tabular}

Toute trace de la consonne historique du latin est donc phonétiquement effacée au pluriel, ce qui permet d'expliquer une seule réalisation, au sein de l'aquitanopyrénéen (pour ce qui concerne l'occitan). Nous pouvons donc définir une graduation supplémentaire de tolérance des finales. L'Ouest du gascon sera plus permissif et acceptera la forme [dus] (où le phonème /1/ vocalisé dans la réalisation [dews] puis réduit à [dus] est bien présent) et la zone Est qui ne l'acceptera pas, comme le démontre la carte 2463 de l'Atlas Linguistique de la Gascogne (ALG).

L'étude devant attaque en voyelle va permettre d'affiner encore cette graduation. En effet, dans ce contexte, la zone Sud-Est de la Gascogne va conserver son obstruante finale comme l'indique la synthèse de la carte 2462 de l'ALG ci-dessous, réalisée par mes soins.

(9) Synthèse carte 2462 ALG 


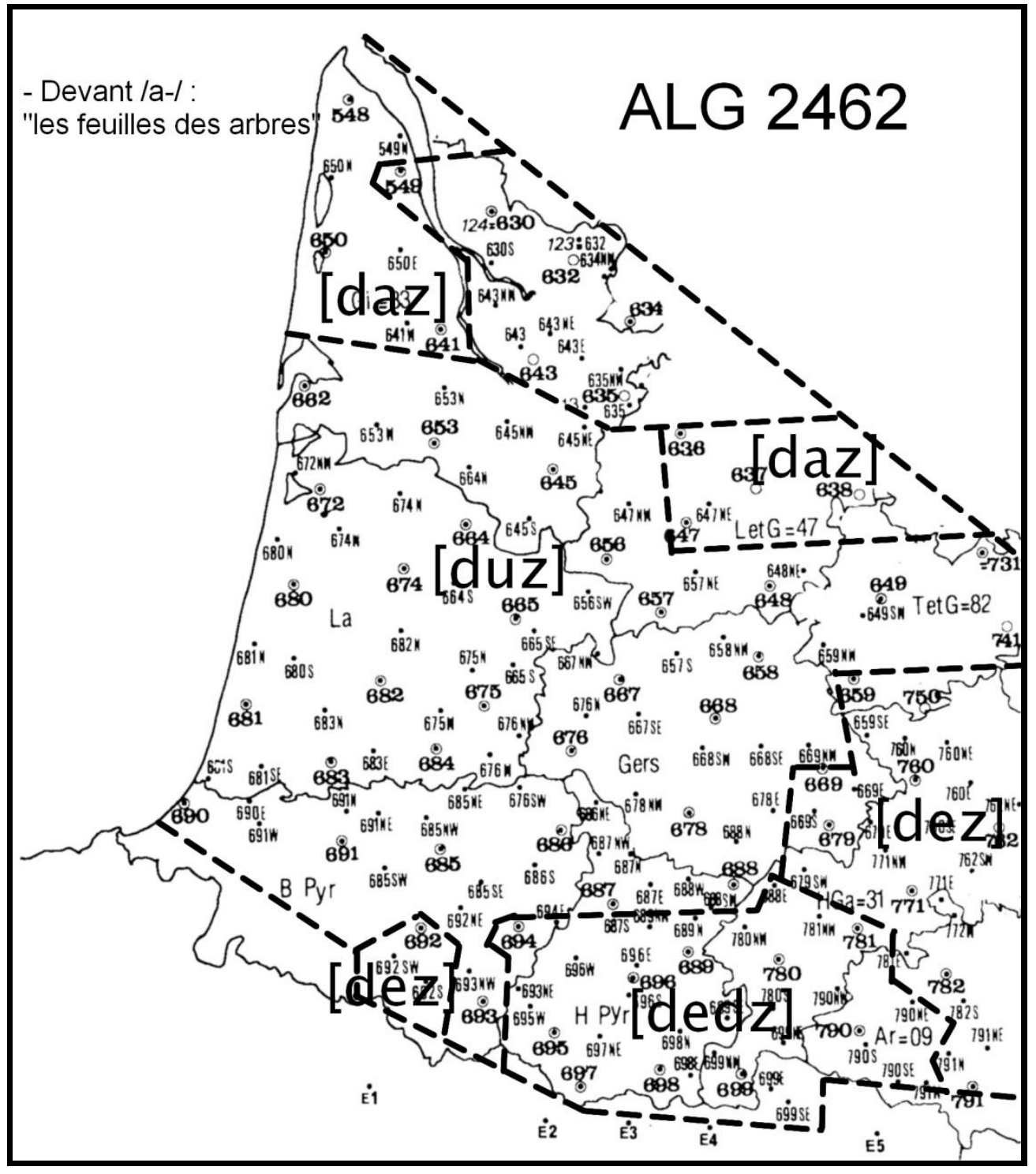

Une différence importante apparaît devant voyelle dans la partie Sud en zone de l'article eth.

(10) Réalisation en sandhi en aquitanopyrénéen devant voyelle 


\begin{tabular}{|c|c|c|c|}
\hline \multicolumn{4}{|c|}{ Devant voyelle } \\
\hline $\begin{array}{l}\text { Article défini } \\
\text { singulier }\end{array}$ & $\begin{array}{l}\text { Article défini }+ \\
\text { morphème } \\
\text { pluriel }\end{array}$ & Contraction singulier & $\begin{array}{l}\text { Contraction }+ \\
\text { morphème pluriel }\end{array}$ \\
\hline le [le] & les [les] & $\begin{array}{l}\text { G.or : deu }[\mathrm{du}] \\
\text { L.occ : del [del] }\end{array}$ & \multirow{2}{*}{$\begin{array}{l}\text { deus } \\
\text { dels } \\
\text { [dez] }\end{array}$} \\
\hline lo $[\mathrm{lu}]$ & los [lus] & $\operatorname{del}[\mathrm{del}]$ & \\
\hline eth [et] & eths [es] & $\operatorname{deth}[\operatorname{det}]$ & $\begin{array}{l}\text { deths } \quad[\text { dedz }] \\
([\operatorname{dez}])\end{array}$ \\
\hline
\end{tabular}

Nous pouvons donc identifier trois traitements devant voyelle sur la partie occitane de l'aquitanopyrénéen, alors qu'il n'y en avait que deux devant consonne, dont la synthèse globale est la suivante :

- La zone Ouest est la plus permissive et conserve systématiquement le phonème consonantique étymologique de l'article [dus]/[duz], aussi bien devant consonne que voyelle.

- La zone Sud, intermédiaire, le conserve généralement devant voyelle et jamais devant consonne $[\mathrm{dedz}]([\mathrm{dez}]) /([\mathrm{des}]$.

- $\quad$ La zone Est qui ne le conserve jamais [des]/[dez].

Cet élément affine les traits qui rapprochent le gascon oriental du languedocien occidental, et contribue à expliquer une parfaite intercompréhension, quand de nombreux traits phonétiques les opposent et rapprochent le gascon oriental du gascon occidental.

Cela permet également d'expliquer toutes les réalisations des contractions avec d'autres prépositions du gascon toulousain d'une part, mais également les réalisations du français méridional, superstrat, d'autre part. 
(11) Réalisation en sandhi en gascon oriental

\begin{tabular}{|l|l|l|}
\hline Préposition & Contraction singulier & Contraction pluriel \\
\hline ende [end'e] (pour) & endeu [end'u] & endeus [end'es] [end'ej] \\
\hline entà [ent'a] (chez) & entau [ent'aw] & entaus [ent'as] [ent'aj] \\
\hline per [per] (par) & peu [pu] & peus [pes] [pej] \\
\hline a [a] (à) & au [au] & as [as] [aj] \\
\hline
\end{tabular}

La yodisation ([dej]/[di], [entaj], etc.) n'est qu'un trait phonétique de sonorisation par assimilation régressive. Elle occupe par conséquent aussi bien la position de coda. Les phones [s] et [j], dans ce cas, sont deux morphèmes du pluriel en distribution complémentaire avec contrainte sourde ou sonore, phénomène détaillé par Lieutard (2004 2).

Avec un système syllabique plus permissif, comme celui du gascon non oriental typique de l'aquitanopyrénéen, nous aurons les formes deus [dus]. Au contraire en provençal, typique de l'arvernoméditerranéen, nous aurons un format moins permissif avec des formes en dels [de]. Ainsi, cette hypothèse permet d'expliquer la variation en finale en sandhi sur la totalité du territoire occitan et d'affiner ainsi la graduation de tolérance des finales au sein de l'aquitanopyrénéen proposée par Bec (1963). 
ALIBERT, L. (2000). Gramatica occitana, segon los parlars lengadocian. Barcelona : I.E.O.-I.E.C,

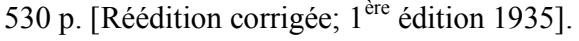

BEC, P. (1995). La langue occitane. Paris : PUF, Coll. Que sais-je ?

BEC, P. (1973). Manuel pratique d'occitan moderne. Paris : coll. Connaissance des langues.

CHABBERT, R. (1959) Quelques remarques de phonétique : s final. Revue du Tarn, 277 -279. Albi.

COSTA, G. (1986). Atlas linguistique « Sacaze » des confins catalano-languedociens. Saint-Estève : P. Société des professeurs de catalan.

DELL, F. (1995). Consonant clusters and phonological syllables in French. Lingua, $n^{\circ}$ 95. 5-26.

ENSERGUEIX, J. (2012). Grammaire des parlers occitans du Couserans. Ariège : IEO Ariège.

GILLIÉRON, J. et EDMONT, E. (1968). Atlas linguistique de la France. Bologna : Champion.

KAYE, J. \& LOWENSTAMM J. (1984). De la syllabicité. F.Dell, D.Hirst \& J.-R. Vergnaud (eds), Forme sonore du langage, 123-159. Paris : Hermann.

LIEUTARD, H. (2004 - 1). Constrenchas fonologicas e morfologia del plural. Linguistica Occitana 1, revue électronique de linguistique occitane. (www.revistadoc.org).

LIEUTARD, H. (2004 - 2). Spécificité morphologique du pluriel languedocien: la notion de « cheville ». Cahiers de grammaire 29, Questions de linguistique et de dialectologie romane, 89 104.Université de Toulouse le Mirail, laboratoire ERSS.

LIEUTARD, H. (2000). Phonologie et morphologie du parler occitan de Graulhet (Tarn) : structure, contenu et rôle de la syllabe. Thèse de Doctorat. Université de Montpellier III.

MAURAND, G. (1974). Phonétique et phonologie du parler occitan d'Ambialet (Tarn). Toulouse : EUS.

NÈGRE, E. (1978). L'article et pronom toulousain Lé. Mélanges de philologie romane offerts à Charles Camproux, vol. 2, 960-5/1197. Montpellier : Centre d'estudis occitans.

POJADA, P. (2009). Répertoire toponymique des communes de la région Midi-Pyrénées. Portèth de Garona : Loubatières.

ROHLFS, G. (1977). Le gascon : étude de philologie pyrénéenne. Pau : Marrimpouey jeune. [3 $3^{\text {ème }}$ éd.]

RONJAT, J. (1930-41). Grammaire historique des parlers provençaux modernes. Montpellier: Société des langues romanes.

ROQUE-FERRIER, A. (1879). Vestiges d'un article archaïque roman conservés dans les dialectes du midi de la France. Bas-Languedoc et Provence, Revue des langues romanes, $n^{\circ} 16,114-139$.

ROQUE-FERRIER, A. (1876). De la double forme de l'article et des pronoms en langue d'oc. BasLanguedoc et Provence, Revue des langues romanes $n^{\circ} 9$ (2.1), 125-137.

SAUZET, P. et BRUN-TRIGAUD G. (2014). L' aso e l' òmi : una paradòxa de cronologia relativa en gascon. Actes du Xème congrès de l'AIEO (Association Internationale des Études Occitanes), Juin 2011, 486-505. Béziers : Lambert-Lucas, LOS QUE FAN VIURE E TRESLUSIR L'OCCITAN.

SAUZET, P. (2004). Variation des finales occitanes et format de la syllabe. Nouveaux départs en phonologie : les conceptions sub- et suprasegmentales, 33-48. Trudel Meisenburg \& Maria Selig eds, Tübingen, Gunter Narr.

SAUZET, P. (1993). Attenance, gouvernement et mouvement en phonologie. Les constituants dans la phonologie et la morphologie de l'occitan. Montpellier: CEO/UPV 505 p.

SAUZET, P. (1974). Approches de la phonologie et de la morphologie d'un parler occitan: le dialecte de Sumène. Gard : Mémoire de maîtrise, Université de Poitiers.

SEGUY, J. (1973). Atlas linguistique et ethnographique de la Gascogne. Paris : CNRS. 
SUMIEN, D. (2009). Classification des dialectes occitans. Linguistica Occitana 7. (www.revistadoc.org).

\footnotetext{
${ }^{\mathrm{i}}()_{\sigma}$ note les limites de syllabes, es signifie extrasyllabique et l'effacement des segments sans statut légitime est noté en le barrant.

ii Toulousain, Grisolles, pays de Foix avec Pamiers, Foix, le Mas-d'Azil, la Bastide-de-Sérou, Massat, Auzat, Mérens, etc...
} 